\title{
茧火虫氨基氧化荧光素相似物作为有机发光二极管材料的理论研究
}

\author{
闵春刚 $*, a$ 李作盛 ${ }^{b}$ 崔小英 ${ }^{a}$ 杨喜昆 ${ }^{a}$ \\ 黄绍军 ${ }^{a}$ 王绍华 $a$ 任爱民*,b \\ $\left({ }^{a}\right.$ 昆明理工大学分析测试研究中心 昆明 650093) \\ ( ${ }^{b}$ 吉林大学理论化学研究所 理论化学计算国家重点实验室 长春 130023)
}

\begin{abstract}
摘要 由于萤火虫荧光素具有发光效率高, 能发出从绿色到红色多种颜色光的特点使其在有机发光器件领域存在巨大 的应用潜力. 为了探索结构和性质的关系, 我们用苯并吡喃、联苯、联吡啶、菲、邻二氮杂菲、芴和晕苯取代氧化荧 光素中的苯并噻唑部分, 设计了一系列氨基氧化苂光素相似物. 本工作用密度泛函理论和含时密度泛函理论方法对这 些化合物进行了一系列光电性质的研究. 计算结果表明, 含有氮原子的氨基联吡啶氧化苂光素(BPAOL)和氨基邻二氮 杂菲氧化苂光素(PMAOL)与含有碳原子的氨基联苯氧化荧光素(BIAOL)和氨基菲氧化苂光素(PHAOL)比较具有较小的 最高占据分子轨道(HOMO)和最低非占据分子轨道(LUMO)轨道能量及更大的电离能(IP)和电子亲和势(EA)值. 化合物 氨基氧化荧光素(AOL)，BIAOL，BPAOL，PHAOL，PMAOL，氨基葋氧化荧光素(FLAOL)和氨基晕苯氧化荧光素 (COAOL) 可以作为电子注入/传输材料. AOL、PHAOL、FLAOL 和 COAOL 可以作为蓝色发光材料.
\end{abstract}

关键词 电离能和电子亲和势; 重组能; 氧化苂光素相似物; 发射光谱; 含时密度泛函理论

\section{Theoretical Investigation on Firefly Oxyluciferin Analogs Bearing an Amino Group Used as Organic Light-Emitting Diodes Materials}

\author{
Min, Chungang ${ }^{*, a}$ \\ Li, Zuosheng ${ }^{b}$ \\ Cui, Xiaoying ${ }^{a}$ \\ Yang, Xikun ${ }^{a}$ \\ Huang, Shaojun ${ }^{a} \quad$ Wang, Shaohua ${ }^{a} \quad$ Ren, Aimin ${ }^{*, b}$ \\ $\left({ }^{a}\right.$ Research Center for Analysis and Measurement, Kunming University of Science and Technology, Kunming 650093) \\ $\left({ }^{b}\right.$ State Key Laboratory of Theoretical and Computational Chemistry, Institute of Theoretical Chemistry,
}

Jilin University, Changchun 130023)

\begin{abstract}
Firefly oxyluciferin has great potential application in organic light-emitting devices, because of the high efficiency and wide range of multicolor light from green to red. To gain an insight into the structure-property relationships, a set of firefly oxyluciferin analogs bearing an amino group with benzopyran, biphenyl, bipyridine, phenanthrene, phenanthroline, fluorine and coronene instead of benzothiazole ring were designed. In this study, a systematic investigation into them was carried out using the density functional theory and time-dependent density functional theory methods. The calculated values show that bipyridylaminooxyluciferin (BPAOL) and orthophenanthrolylaminooxyluciferin (PMAOL) with nitrogen atom have smaller the highest occupied molecular orbital (HOMO) and the lowest unoccupied molecular orbital (LUMO) energies, larger ionization potential (IP) and electron affinity (EA) values than biphenylaminooxyluciferin (BIAOL) and phenanthrylaminooxyluciferin (PHAOL) with carbon atom. Compound aminooxyluciferin (AOL), BIAOL, BPAOL, PHAOL, PMAOL, fluorenylaminooxyluciferin (FLAOL) and coronenylaminooxyluciferin (COAOL) can be used as electron-injection/transporting materials. AOL, PHAOL, FLAOL and COAOL can be used as blue light-emitting materials. These results indicate that the firefly
\end{abstract}

\footnotetext{
*E-mail: minchungang@163.com, aimin_ren@yahoo.com

Received September 3, 2014; revised October 13, 2014; published online October 21, 2014.

Project supported by the National Natural Science Foundation of China (Nos. 21473071, 21173099, 21363012, 51363012, 51374117), the National Key Basic Research Program (973 Program, No. 2013CB834801), the Special Funding to Basic Scientific Research Projects for Central Colleges, the Applied Basic Research Plans Program of Yunnan Province (No. 2011FZ040), the Scientific Research Fund of Yunnan Provincial Education Department (No. 2012Y545), the Training Foundation for Talents of Kunming University of Science and Technology (No. KKSY201232040) and the Foundation of State Key Laboratory of Theoretical and Computational Chemistry.

国家自然科学基金(Nos. 21473071, 21173099, 21363012, 51363012, 51374117)、国家重点基础研究发展规划(973 计划, No. 2013CB834801)、高校基础 科学研究专项、云南省应用基础研究计划(No. 2011FZ040)、云南省教育厅科学研究基金(No. 2012Y545)、昆明理工大学人才培养基金(No. KKSY201232040)和吉林大学理论化学计算国家重点实验室开放课题基金资助项目.
} 
oxyluciferin analogs bearing an amino group have many interesting properties and are good candidates for optoelectronic application.

Keywords ionization potential and electron affinity; reorganization energy; oxyluciferin analogs; emission spectrum; time-dependent density functional theory

在过去的几十年里, 有机发光二极管(OLEDs)由于 具有响应快速、对比度高、分辨率高、工作电压低、颜 色可调、稳定性好等优点使其在平板显示和照明设备中 表现出良好的应用前景 ${ }^{[1 \sim 7]}$. 而荧光材料由于具有发光 效率高、电荷传输能力好、使复杂工艺简单化等特点大 量应用于 OLED 器件 ${ }^{[8]}$. 其中同时拥有电子供体和电子 受体部分的发光物质由于存在分子内电荷转移, 使其具 有独特的光电性质, 因此成为 OLED 器件优良的备选材 料 ${ }^{[0 ~ 13]}$. 但是, 具有分子内电荷转移特点的材料一般发 光效率都很低, 很难满足 OLED 器件的要求 ${ }^{[14]}$. 而在自 然界中, 具有分子内电荷转移特点的萤火虫生物发光底 物氧化荧光素的发光效率却高达 $(41.0 \pm 7.4) \%^{[15 \sim 17]}$, 我 们在以前的研究中发现其可以同时作为蓝光材料和电 子传输材料 ${ }^{[18]}$. 然而, 氧化苂光素的生物发光光谱对盐 浓度 ${ }^{[19]}$ 及 $\mathrm{pH}^{[20,21]}$ 等环境因素非常敏感, 这一特点无论 对生物发光体系还是 OLED 器件而言都是不受欢迎的. 2010 年, Takakura 等 ${ }^{[22]}$ 用氨基取代苯并噻唑环上的羟 基, 同时分别用喹啉、菜、香豆素取代苯并噻唑环合成 了氨基荧光素 $(A L)$ 、氨基喹啉苂光素 $(\mathrm{QAL})$ 、氨基荎苂 光素(NAL)和氨基香豆素荧光素(CAL)四种苂光素相似 物, 并研究了它们的生物发光和苂光性质. 结果表明, 它们和萤火虫苂光酶作用可以发出红、黄、绿等不同颜 色的光, 且 CAL 的苂光光谱受溶剂极性的影响很小, 在 二甲亚砜、氯仿、苯、甲醇等溶剂中的发射波长基本保 持不变. 2012 年, 我们 ${ }^{[23]}$ 用两个更大的基团葱和芘取代 苯并噻唑环进而设计了两种新的氨基苂光素相似物, 氨 基葱苂光素(AAL)和氨基萠苂光素(PAL), 进而研究了 AL, QAL, NAL, CAL, AAL, PAL 及对应的酮式氧化荧 光素相似物的苂光性质, 计算结果表明 AAOL 的发射光 谱和天然萤火虫的发射光谱比较接近.

受上述报道鼓励, 本文中我们用苯并吡喃、联苯、 联吡啶、菲、邻二氮杂菲、芴和晕苯取代苯并噻唑环进 而设计了 7 种新的氨基氧化苂光素相似物, 氨基苯并吡 喃氧化苂光素(BEAOL)、氨基联苯氧化苂光素(BIAOL)、 氨基联吡啶氧化苂光素(BPAOL)、氨基菲氧化荧光素 (PHAOL)、氨基邻二氮杂菲氧化苂光素(PMAOL)、氨基 芴氧化苂光素 (FLAOL) 和氨基军苯氧化苂光素 (COAOL)(图 1). 进而研究了这些氨基氧化苂光素相似 物的电离能、电子亲和势、空穴抽取能、电子抽取能等 光电性质, 最终评估了它们各自作为 OLED 材料的可能 性.

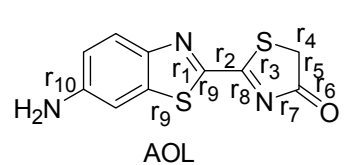<smiles></smiles><smiles>Nc1ccc(-c2ccc(C3=NC(=O)CS3)cc2)cc1</smiles><smiles>Nc1ccc(-c2ccc(C3NC(=O)CS3)nc2)nc1</smiles>
BIAOL

BPAOL
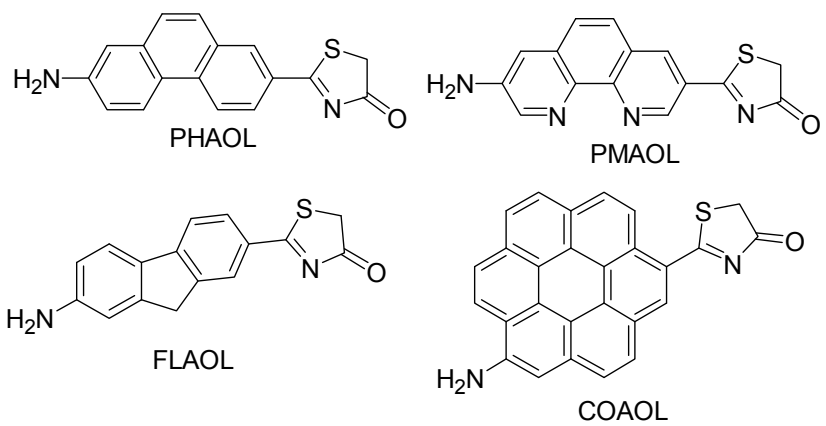

图 1 萤火虫氨基氧化苂光素相似物 AOL, BEAOL, BIAOL, BPAOL, PHAOL, PMAOL, FLAOL 和 COAOL 的化学结构

Figure 1 Chemical structures of firefly oxyluciferin analogs bearing an amino group AOL, BEAOL, BIAOL, BPAOL, PHAOL, PMAOL, FLAOL and COAOL

\section{1 结果与讨论}

\section{1 基态几何结构}

MPW3PBE/6-31+G $(d)$ 方法优化得到的氨基氧化苂 光素衍生物 AOL, BEAOL, BIAOL, BPAOL, PHAOL, PMAOL, FLAOL 和 COAOL 在中性几何构型下的重要 键长和二面角值列于表 1 中. 由表 1 可见, 苯并吡喃、 联苯、联吡啶、菲、邻二氮杂菲、芴和晕苯的取代对 $r_{2} \sim$ $r_{8}$ 和 $r_{10}$ 键长几乎没有影响. 变化最大的是 $r_{1}$ 和 $r_{9}$ 键, 由 于氮原子被碳原子取代 $r_{1}$ 伸长了 $0.009 \sim 0.021 \mathrm{~nm}$. 而 $r_{9}$ 由于硫原子被碳原子取代缩短了 $0.034 \sim 0.041 \mathrm{~nm}$. 此 外两个片段之间的二面角差别很小, 均接近 $180^{\circ}$, 由此 说明这些氨基氧化苂光素化合物的平面性很好.

\section{2 前线分子轨道}

因为分子的电荷传输性质与最高占据分子轨道 (HOMO) 和最低非占据分子轨道(LUMO)的能量及电子 云分布相关, 对分子的 HOMO、LUMO 轨道及 HOMO 和 LUMO 轨道间能隙 $\left(\Delta \mathrm{E}_{\mathrm{H}-\mathrm{L}}\right)$ 的研究显得十分必要 ${ }^{[24]}$. 图 2 给出了 MPW3PBE/6-31+ G $(d)$ 方法得到的所研究分 子的 HOMO 和 LUMO 轨道电子云密度分布图. 表 2 给 出了不同片段对 HOMO 和 LUMO 轨道的贡献. 由图 2 
表 1 MPW3PBE/6-31+G(d)方法得到的中性 AOL, BEAOL, BIAOL, BPAOL, PHAOL, PMAOL, FLAOL 和 COAOL 的一些重要键 长 $(\mathrm{nm})$ 和二面角 $\left(^{\circ}\right)$

Table 1 Selected important bond lengths $(\mathrm{nm})$ and dihedral angles $\left(^{\circ}\right)$ of AOL, BEAOL, BIAOL, BPAOL, PHAOL, PMAOL, FLAOL and COAOL in neutral state obtained by MPW3PBE/6-31+ $\mathrm{G}(d)$ method

\begin{tabular}{ccccccccc}
\hline Compd. & AOL & BEAOL & BIAOL & BPAOL & PHAOL & PMAOL & FLAOL & COAOL \\
\hline $\mathrm{r}_{1}$ & 0.130 & 0.151 & 0.140 & 0.140 & 0.139 & 0.139 & 0.141 & 0.145 \\
$\mathrm{r}_{2}$ & 0.145 & 0.145 & 0.146 & 0.146 & 0.146 & 0.146 & 0.146 & 0.148 \\
$\mathrm{r}_{3}$ & 0.177 & 0.178 & 0.178 & 0.178 & 0.178 & 0.178 & 0.178 & 0.178 \\
$\mathrm{r}_{4}$ & 0.181 & 0.180 & 0.180 & 0.180 & 0.180 & 0.180 & 0.180 & 0.180 \\
$\mathrm{r}_{5}$ & 0.154 & 0.154 & 0.154 & 0.154 & 0.154 & 0.154 & 0.154 & 0.154 \\
$\mathrm{r}_{6}$ & 0.121 & 0.121 & 0.121 & 0.121 & 0.121 & 0.121 & 0.121 & 0.121 \\
$\mathrm{r}_{7}$ & 0.140 & 0.139 & 0.139 & 0.140 & 0.139 & 0.140 & 0.139 & 0.139 \\
$\mathrm{r}_{8}$ & 0.130 & 0.130 & 0.130 & 0.129 & 0.130 & 0.129 & 0.130 & 0.130 \\
$\mathrm{r}_{9}$ & 0.176 & 0.135 & 0.141 & 0.141 & 0.142 & 0.142 & 0.141 & 0.139 \\
$\mathrm{r}_{10}$ & 0.138 & 0.139 & 0.139 & 0.138 & 0.139 & 0.138 & 0.139 & 0.139 \\
$\angle \mathrm{CCCN}^{a}$ & 179.9 & 179.9 & 179.9 & 178.8 & 179.9 & 179.5 & 180.0 & 179.8 \\
\hline
\end{tabular}

${ }^{a}$ The dihedral angle is $\angle \mathrm{NCCN}$ in AOL.

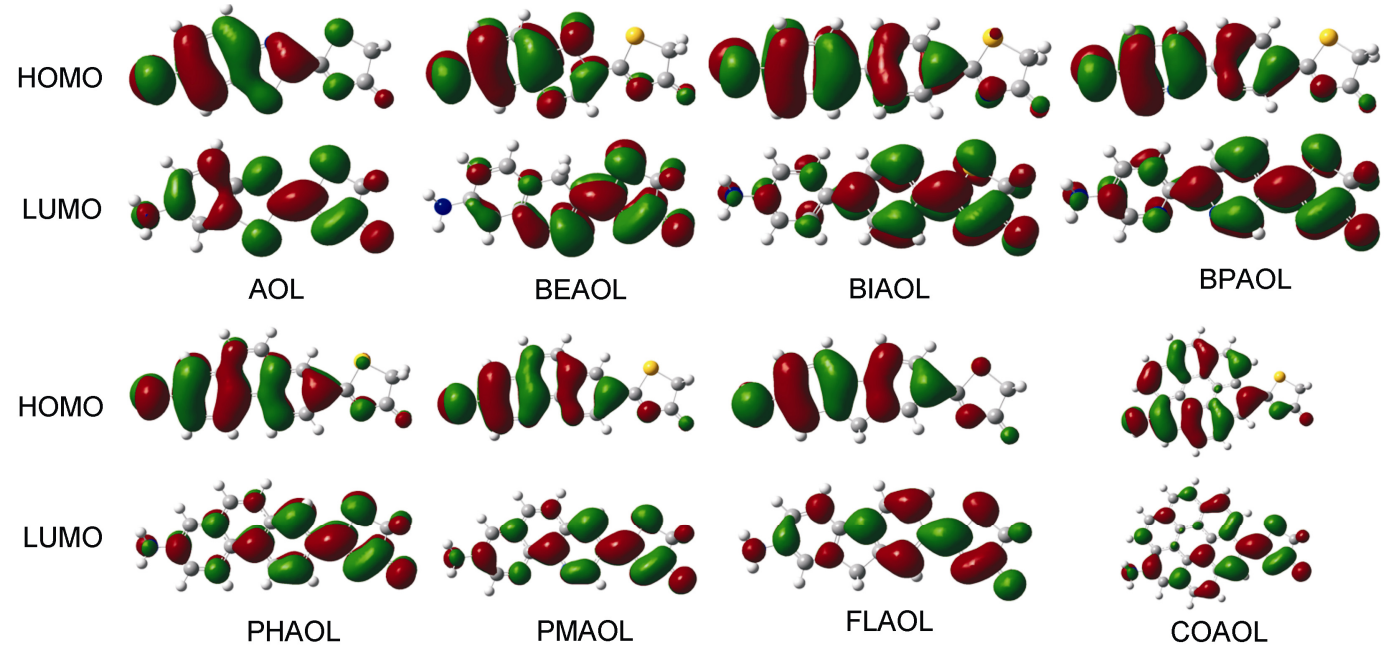

图 2 MPW3PBE/6-31+G(d)得到的 AOL, BEAOL, BIAOL, BPAOL, PHAOL, PMAOL, FLAOL 和 COAOL 的前线轨道示意图 Figure 2 Plots of the frontier orbitals for AOL, BEAOL, BIAOL, BPAOL, PHAOL, PMAOL, FLAOL and COAOL by MPW3PBE/ $6-31+\mathrm{G}(d)$

表 2 AOL, BEAOL, BIAOL, BPAOL, PHAOL, PMAOL, FLAOL 和 COAOL 中不同片段对 HOMO 和 LUMO 轨道贡献的百分比(\%)

Table 2 Contribution of electron density (\%) for AOL, BEAOL, BIAOL, BPAOL, PHAOL, PMAOL, FLAOL and COAOL

\begin{tabular}{|c|c|c|c|c|c|c|}
\hline \multirow{2}{*}{ Compd. } & \multicolumn{2}{|c|}{$\mathrm{R}$ group } & \multicolumn{2}{|c|}{ Thiazoline ring } & \multicolumn{2}{|c|}{ Carbonyl oxygen } \\
\hline & HOMO & LUMO & HOMO & LUMO & HOMO & LUMO \\
\hline $\mathrm{AOL}$ & 95.79 & 64.60 & 3.75 & 30.72 & 0.46 & 4.67 \\
\hline BEAOL & 97.77 & 55.72 & 1.88 & 39.38 & 0.35 & 4.90 \\
\hline BIAOL & 99.30 & 92.80 & 0.62 & 6.24 & 0.08 & 0.96 \\
\hline BPAOL & 99.17 & 95.54 & 0.75 & 3.92 & 0.08 & 0.54 \\
\hline PHAOL & 96.53 & 55.21 & 2.94 & 38.56 & 0.53 & 6.23 \\
\hline PMAOL & 98.38 & 65.90 & 1.37 & 29.30 & 0.24 & 4.79 \\
\hline FLAOL & 96.09 & 64.14 & 3.38 & 31.10 & 0.53 & 4.77 \\
\hline COAOL & 97.88 & 81.16 & 1.65 & 16.43 & 0.47 & 2.41 \\
\hline
\end{tabular}

和表 2 可见, 所有化合物的 HOMO 轨道电子云主要位于 $\mathrm{R}$ 基团上, 噻唑环和羰基氧对轨道的贡献很小. 对于 LUMO 轨道, 除 BIAOL 和 BPAOL 外, R 基团对 LUMO 轨道的贡献与 HOMO 轨道比较大幅减小, 如在 BEAOL
中，氨基苯并吡喃基团对 $\mathrm{HOMO}$ 轨道的贡献高达 $97.77 \%$, 而对 LUMO 轨道的贡献仅有 $55.72 \%$. 相对而 言, 噻唑环和羰基氧对 LUMO 轨道的贡献增加, 分别为 $39.38 \%$ 和 4.90\%. 也就是说, 从 HOMO 到 LUMO 轨道 
的跃迁过程中, 有一部分电子从 $\mathrm{R}$ 基团转移到噻唑环和 羰基氧上. 而在 BIAOL 和 BPAOL 中, 各片段对 LUMO 轨道的贡献与 $\mathrm{HOMO}$ 轨道比较变化不大, 也就是说, 从 HOMO 到 LUMO 的跃迁过程中, 几乎没有电子转移, 这可能是由于 BIAOL 的两个苯环及 BPAOL 的两个吡 啶环之间存在一个碳碳单键的缘故.

所研究化合物的 HOMO 和 LUMO 轨道能量值及能 隙 $\left(E_{\mathrm{HOMO}} \sim E_{\mathrm{LUMO}}\right)$ 列于表 3 中. 理论上讲, HOMO 轨道 能量越高, 越容易失去电子产生空穴使分子具有好的空 穴注入能力. 相反, LUMO 轨道能量越低, 越容易接受 电子使分子具有好的电子注入能力. 如表 3 所示, BPAOL 和 PMAOL 分别与 BIAOL 和 PHAOL 比较, 发 现氮原子对碳原子的取代使分子的空穴注入能力减弱, 电子注入能力增强, 同时使能隙变大, 不利于阳离子的 形成. 在所研究的氨基氧化苂光素相似物中, COAOL 具有最高的 HOMO 能量 $(-5.76 \mathrm{eV})$, 因此具有最强的 空穴注入能力, 但其 HOMO 能量值仍低于具有多重功 能的 BNPB $(-5.34 \mathrm{eV})^{[25,26]}$, 说明所有氨基氧化苂光素 衍生物都不适合作为空穴传输材料. 相对的, 它们都拥 有比多重功能的 BNPB $(-2.03 \mathrm{eV})^{[25,26]}$ 低的 LUMO 轨 道能量, 因此具有较强的电子注入能力. 与 AOL 的能隙 比较发现, BEAOL, BIAOL, BPAOL, PHAOL, PMAOL, FLAOL 的能隙均增大, 只有 COAOL 的能隙减小, 说明 大的刚性基团晕苯的引入有利于阴、阳离子的形成. 总 之, 通过用苯并吡喃、联苯、联吡啶、菲、邻二氮杂菲、 芴和晕苯取代苯并噻唑环可以调节这些氨基氧化苂光 素衍生物的光学性质.

\section{3 电离能、电子亲和势和重组能}

为了评估空穴和电子注入的能垒, 我们研究了电离
能(IP)和电子亲和势(EA), 电离能越小, 空穴的注入就 越容易; 而电子亲和势越大，电子的注入就越容易. MPW3PBE $/ 6-31+\mathrm{G}(d)$ 方法计算的 IPs 和 EAs 值列于表 4 中. 如表 4 所示, 所研究化合物绝热电离能和绝热电 子亲和势的顺序分别为 $\mathrm{COAOL}<\mathrm{FLAOL}<\mathrm{BIAOL}<$ PHAOL $<$ BEAOL $<$ BPAOL $<$ AOL $<$ PMAOL 和 $\mathrm{PMAOL}>\mathrm{AOL}>\mathrm{COAOL}>\mathrm{BPAOL}>\mathrm{PHAOL}>$ BIAOL $>$ FLAOL $>$ BEAOL. 化合物 BPAOL 和 PMAOL 的 IP 和 EA 值与化合物 BIAOL 和 PHAOL 比较, 与第 1.2 节中对 HOMO 和 LUMO 轨道能量讨论的结果相同, 说明氮原子对碳原子的取代可以调节电子传输能力. 所 有化合物的 $\operatorname{IP}(a)$ 值均大于好的多功能材料 BNPB 的电 离能 $(6.37 \mathrm{eV})^{[25,26]}$, 说明这些衍生物不适合作为空穴传 输材料. 另外, 与实验上已证实的好的多功能材料 BNPB 比较(EA: $1.16 \mathrm{eV})^{[25,26]}$ 发现, 除 BEAOL (EA: 1.06 $\mathrm{eV}$ )外，所有化合物的电子传输性能都比 BNPB 好，即 可作为电子传输材料, 其中 PMAOL 的电子亲和势最大 为 $1.71 \mathrm{eV}$.

最后, 为了定性地表示空穴和电子迁移速率的快 慢, 我们又计算了空穴重组能 $\left(\lambda_{\text {hole }}\right)$ 和电子重组能 $\left(\lambda_{\text {electron }}\right)^{[27,28]}$. 如表 4 所示, 除 BEAOL 外, 所有化合物的 $\lambda_{\text {electron }}$ 均小于 $\mathrm{AOL}$, 说明苯并噻唑环被联苯、联吡啶、 菲、邻二氮杂菲、芴和晕苯取代后, 它们的电子传输速 率均加快. 除 BEAOL 外, 所有衍生物的 $\lambda_{\text {electron }}$ 和 $\lambda_{\text {hole }}$ 之间的差都小于 AOL, 表明这些化合物的空穴和电子 注入的平衡性得到了很大提高.

\section{4 电子吸收和发射光谱}

表 5 列出了 TD MPW3PBE $/ 6-31+\mathrm{G}(d)$ 方法在基态 优化几何基础上得到的所研究化合物的吸收光谱、振子

表 3 所研究化合物的 HOMO, LUMO 轨道能量及能隙

Table 3 HOMO, LUMO energies and HOMO-LUMO gaps of studied compounds

\begin{tabular}{lcccrrrrr}
\hline Compd. & AOL & BEAOL & BIAOL & BPAOL & PHAOL & PMAOL & FLAOL & COAOL \\
\hline$E_{\mathrm{HOMO}} / \mathrm{eV}$ & -6.23 & -6.09 & -5.95 & -6.29 & -6.00 & -6.36 & -5.80 & -5.76 \\
$E_{\mathrm{LUMO}} / \mathrm{eV}$ & -2.90 & -2.33 & -2.55 & -2.78 & -2.55 & -2.79 & -2.46 & -2.68 \\
$E_{\mathrm{HOMO}} \sim E_{\mathrm{LUMO}} / \mathrm{eV}$ & 3.33 & 3.76 & 3.40 & 3.51 & 3.45 & 3.57 & 3.34 & 3.08 \\
\hline
\end{tabular}

表 4 所研究化合物的电离能、电子亲和势、抽取能和重组能 $(\mathrm{eV})$

Table 4 Ionization potentials, electron affinities, extraction potentials and reorganization energies for studied compounds $(\mathrm{eV})$

\begin{tabular}{ccccccccc}
\hline Compd. & IP $(v)$ & IP $(a)$ & HEP & EA $(v)$ & EA $(a)$ & EEP & $\lambda_{\text {hole }}$ & $\lambda_{\text {electron }}$ \\
\hline AOL & 7.85 & 7.60 & 7.45 & 1.41 & 1.66 & 1.91 & 0.40 & 0.50 \\
BEAOL & 7.69 & 7.45 & 7.27 & 0.78 & 1.06 & 1.35 & 0.42 & 0.57 \\
BIAOL & 7.48 & 7.22 & 7.07 & 1.13 & 1.34 & 1.54 & 0.41 & 0.41 \\
BPAOL & 7.84 & 7.58 & 7.43 & 1.36 & 1.59 & 1.81 & 0.41 & 0.45 \\
PHAOL & 7.46 & 7.24 & 7.11 & 1.18 & 1.38 & 1.58 & 0.35 & 0.40 \\
PMAOL & 7.86 & 7.64 & 7.51 & 1.41 & 1.71 & 1.83 & 0.35 & 0.42 \\
FLAOL & 7.27 & 7.05 & 6.90 & 1.08 & 1.27 & 1.46 & 0.37 & 0.38 \\
COAOL & 6.99 & 6.82 & 6.70 & 1.48 & 1.63 & 1.78 & 0.29 & 0.30 \\
\hline
\end{tabular}


表 5 TD MPW3PBE/6-31+G(d)方法计算得到的 AOL, BEAOL, BIAOL, BPAOL, PHAOL, PMAOL, FLAOL 和 COAOL 的吸收光 谱、振子强度及主要跃迁组成

Table 5 Absorption spectra oscillator strengths $(f)$ and main configuration obtained by TD MPW3PBE/6-31+G $(d)$ method for AOL, BEAOL, BIAOL, BPAOL, PHAOL, PMAOL, FLAOL and COAOL based on the optimized geometries

\begin{tabular}{|c|c|c|c|c|}
\hline Compd. & Electronic transition & Wavelength/nm & $f$ & Main configuration \\
\hline \multirow[t]{3}{*}{$\mathrm{AOL}$} & $\mathrm{S}_{1}$ & 399.69 & 0.4576 & $\begin{array}{l}\mathrm{HOMO}-1 \rightarrow \text { LUMO }(-0.12027) \\
\mathrm{HOMO} \rightarrow \text { LUMO }(0.69262)\end{array}$ \\
\hline & $\mathrm{S}_{2}$ & 361.09 & 0.0002 & $\begin{array}{l}\text { HOMO }-3 \rightarrow \text { LUMO }(0.69391) \\
\text { HOMO }-2 \rightarrow \text { LUMO }(-0.16412)\end{array}$ \\
\hline & $\mathrm{S}_{3}$ & 340.04 & 0.1482 & $\begin{array}{l}\text { HOMO }-1 \rightarrow \text { LUMO }(0.66800) \\
\text { HOMO } \rightarrow \text { LUMO }(0.11585)\end{array}$ \\
\hline \multirow[t]{3}{*}{ BEAOL } & $\mathrm{S}_{1}$ & 385.92 & 0.0372 & $\mathrm{HOMO} \rightarrow$ LUMO $(0.70404)$ \\
\hline & $\mathrm{S}_{2}$ & 332.66 & 0.0003 & HOMO $-3 \rightarrow$ LUMO $(0.69151)$ \\
\hline & $\mathrm{S}_{3}$ & 313.14 & 0.4206 & HOMO $-1 \rightarrow$ LUMO (0.69490) \\
\hline \multirow[t]{3}{*}{ BIAOL } & $\mathrm{S}_{1}$ & 397.91 & 0.5999 & $\mathrm{HOMO} \rightarrow \mathrm{LUMO}(0.70443)$ \\
\hline & $\mathrm{S}_{2}$ & 340.81 & 0.0004 & $\begin{array}{l}\text { HOMO }-2 \rightarrow \text { LUMO }(0.68797) \\
\text { HOMO }-2 \rightarrow \text { LUMO }+2(-0.11801)\end{array}$ \\
\hline & $\mathrm{S}_{3}$ & 311.30 & 0.0314 & HOMO $-1 \rightarrow$ LUMO (0.68737) \\
\hline \multirow[t]{3}{*}{ BPAOL } & $\mathrm{S}_{1}$ & 385.31 & 0.6284 & $\mathrm{HOMO} \rightarrow \mathrm{LUMO}(0.70424)$ \\
\hline & $\mathrm{S}_{2}$ & 361.34 & 0.0003 & $\begin{array}{l}\mathrm{HOMO}-3 \rightarrow \text { LUMO }(-0.26598) \\
\text { HOMO }-1 \rightarrow \text { LUMO }(0.63955)\end{array}$ \\
\hline & $\mathrm{S}_{3}$ & 345.59 & 0.0003 & $\begin{array}{l}\text { HOMO }-4 \rightarrow \text { LUMO }(0.52319) \\
\text { HOMO }-3 \rightarrow \text { LUMO }(-0.43093) \\
\text { HOMO }-1 \rightarrow \text { LUMO }(-0.11227)\end{array}$ \\
\hline \multirow[t]{3}{*}{ PHAOL } & $\mathrm{S}_{1}$ & 392.00 & 0.5088 & $\begin{array}{l}\mathrm{HOMO}-1 \rightarrow \mathrm{LUMO}+1(-0.13271) \\
\mathrm{HOMO} \rightarrow \mathrm{LUMO}(0.69241)\end{array}$ \\
\hline & $\mathrm{S}_{2}$ & 376.61 & 0.0062 & $\begin{array}{l}\mathrm{HOMO}-1 \rightarrow \text { LUMO }(0.66322) \\
\mathrm{HOMO} \rightarrow \mathrm{LUMO}+1(0.22882)\end{array}$ \\
\hline & $\mathrm{S}_{3}$ & 340.30 & 0.0003 & $\begin{array}{l}\text { HOMO }-3 \rightarrow \text { LUMO }(0.68780) \\
\text { HOMO }-3 \rightarrow \text { LUMO }+2(0.10995)\end{array}$ \\
\hline \multirow[t]{5}{*}{ PMAOL } & $\mathrm{S}_{1}$ & 378.59 & 0.4984 & $\begin{array}{l}\mathrm{HOMO}-1 \rightarrow \mathrm{LUMO}+1(0.13682) \\
\mathrm{HOMO} \rightarrow \mathrm{LUMO}(0.68942)\end{array}$ \\
\hline & & & & HOMO $-5 \rightarrow$ LUMO (0.12495) \\
\hline & $\mathrm{S}_{2}$ & 368.43 & 0.0016 & HOMO $-4 \rightarrow$ LUMO $(0.11451)$ \\
\hline & & & & HOMO $-2 \rightarrow$ LUMO $(0.67711)$ \\
\hline & $\mathrm{S}_{3}$ & 357.95 & 0.0009 & $\begin{array}{l}\mathrm{HOMO}-1 \rightarrow \text { LUMO }(0.63480) \\
\mathrm{HOMO} \rightarrow \mathrm{LUMO}+1(-0.29455)\end{array}$ \\
\hline \multirow[t]{3}{*}{ FLAOL } & $\mathrm{S}_{1}$ & 398.11 & 0.7519 & $\mathrm{HOMO} \rightarrow$ LUMO (0.70352) \\
\hline & $\mathrm{S}_{2}$ & 339.42 & 0.0003 & $\begin{array}{l}\text { HOMO }-2 \rightarrow \text { LUMO }(0.68697) \\
\text { HOMO }-2 \rightarrow \text { LUMO }+2(0.11756)\end{array}$ \\
\hline & $\mathrm{S}_{3}$ & 312.53 & 0.0185 & $\begin{array}{l}\text { HOMO }-4 \rightarrow \text { LUMO }(-0.11427) \\
\text { HOMO }-1 \rightarrow \text { LUMO }(0.67451) \\
\text { HOMO } \rightarrow \text { LUMO }+1(-0.12247)\end{array}$ \\
\hline \multirow[t]{3}{*}{ COAOL } & $\mathrm{S}_{1}$ & 441.96 & 0.4257 & $\begin{array}{l}\mathrm{HOMO}-1 \rightarrow \mathrm{LUMO}+1(0.17350) \\
\mathrm{HOMO} \rightarrow \mathrm{LUMO}(0.68099)\end{array}$ \\
\hline & $\mathrm{S}_{2}$ & 432.43 & 0.0137 & $\begin{array}{l}\mathrm{HOMO}-1 \rightarrow \text { LUMO }(0.61516) \\
\mathrm{HOMO} \rightarrow \mathrm{LUMO}+1(-0.33565)\end{array}$ \\
\hline & $\mathrm{S}_{3}$ & 365.45 & 0.1490 & $\begin{array}{l}\mathrm{HOMO}-1 \rightarrow \text { LUMO }(0.32232) \\
\mathrm{HOMO}-1 \rightarrow \text { LUMO }+2(-0.26384) \\
\mathrm{HOMO} \rightarrow \mathrm{LUMO}+1(0.56075)\end{array}$ \\
\hline
\end{tabular}

强度及主要跃迁组成. 如表 5 所示, AOL, BIAOL, BPAOL, PHAOL, PMAOL, FLAOL 和 COAOL 最大吸收
峰所对应的跃迁都是从 $\mathrm{S}_{0} \rightarrow \mathrm{S}_{1}$, 最大吸收峰分别为 $399.69,397.91,385.31,392.00,378.59,398.11$ 和 441.96 
nm. 化合物 BIAOL, BPAOL, PHAOL, PMAOL, FLAOL 与 $\mathrm{AOL}$ 相比分别蓝移了 $1.78,14.38,7.69,21.1$ 和 1.58 $\mathrm{nm}$. 而 COAOL 则红移了 $42.27 \mathrm{~nm}$. BEAOL 的 $\mathrm{S}_{0} \rightarrow \mathrm{S}_{1}$ 所对应的振子强度很小, 可以说 $\mathrm{S}_{0} \rightarrow \mathrm{S}_{1}$ 是禁阻的, 其最 大振子强度所对应的跃迁为 $\mathrm{S}_{0} \rightarrow \mathrm{S}_{3}$, 与 $\mathrm{AOL}$ 相比最大 吸收峰蓝移 $86.55 \mathrm{~nm}$. 氨基氧化苂光素系列化合物最大 吸收峰顺序为 $\mathrm{BEAOL}<\mathrm{PMAOL}<\mathrm{BPAOL}<\mathrm{PHAOL}<$ $\mathrm{BIAOL}<\mathrm{FLAOL}<\mathrm{AOL}<\mathrm{COAOL}$.

基于优化的激发态几何, 用 TD MPW3PBE/6-31+ $\mathrm{G}(d)$ 方法计算了所研究化合物的发射光谱. 如表 6 所示, AOL, PHAOL, FLAOL 和 COAOL 可以作为蓝色发光材 料, 其发射光谱的电子跃迁是 $\mathrm{S}_{1} \rightarrow \mathrm{S}_{0}$ 态的跃迁, 这些跃 迁主要是由跃迁系数大于 0.68612 的 HOMO 轨道到 LUMO 轨道的跃迁组成的. AOL, PHAOL, FLAOL 和 COAOL 的斯托克位移分别为 32.7, 27.97, 22.61 和 20.26 $\mathrm{nm}$. BEAOL, BIAOL, BPAOL 和 PMAOL 的发射光谱所 对应的振子强度很小, 即所对应的跃迁是禁阻的.

\section{2 结论}

本文主要研究了苯并吡喃、联苯、联吡啶、菲、邻 二氮杂菲、芴和晕苯取代的萤火虫氨基氧化苂光素的结 构和光电性质. 从 IP 和 EA 值来看, 所有氨基氧化苂光 素衍生物的空穴注入能力都很差. 与实验上已证实的好 的多功能材料 BNPB 比较发现, 除 BEAOL 外, 所有化 合物均可作为电子传输材料, 其中 PMAOL 有最大的电 子亲和势为 $1.71 \mathrm{eV}$. 化合物 BPAOL 和 PMAOL 与化合 物 BIAOL 和 PHAOL 比较, 由于氮原子对碳原子的取 代, 使相应的 HOMO 和 LUMO 轨道能量减小, IP 和 EA 值增大, 提高了电子的传输能力. 从吸收光谱上看, 除 BEAOL 外, 所有衍生物对应的跃迁都是从 $\mathrm{S}_{0} \rightarrow \mathrm{S}_{1}$. 此 外，由于基态和激发态间几何结构的改变导致斯托克位 移从 $20.26 \sim 32.7 \mathrm{~nm}$. 这些化合物的发射光谱和振子强 度表明 AOL, PHAOL, FLAOL 和 COAOL 可以作为蓝光
发光材料.

\section{3 计算方法}

近来的研究表明, MPW3PBE ${ }^{[29,30]}$ 泛函能准确地预 测萤火虫氧化苂光素衍生物的光电性质 ${ }^{[18,31]}$. 因此我们 选择 DFT-MPW3PBE 泛函结合 6-31+ G(d) 基组优化氨 基氧化荧光素相似物 AOL, BEAOL, BIAOL，BPAOL， PHAOL, PMAOL, FLAOL 和 COAOL 的基态、阳离子态 和阴离子态的几何结构. 以优化的几何结构为基础, 通 过文献[32, 33]中介绍的方法计算了电离能(IP)、电子亲 和势(EA)、空穴抽取能(HEP)、电子抽取能(EEP)、重组 能( $\lambda$ )、最高占据轨道 $(\mathrm{HOMO})$ 、最低非占据轨道(LUMO) 能量及 HOMO、LUMO 轨道能隙 $\left(\Delta E_{\mathrm{H}-\mathrm{L}}\right)$. 所研究化合物 的第一单重激发态几何通过 TD-DFT//MPW3PBE/6$31+\mathrm{G}(d)$ 方法优化. 在优化基态和激发态几何的基础 上，采用 TD-DFT//MPW3PBE $/ 6-31+\mathrm{G}(d)$ 方法计算了化 合物的吸收和发射光谱. 为了进一步证实所选泛函及基 组的准确性，我们用此方法计算了 $\mathrm{AL}, \mathrm{QAL}, \mathrm{NAL}$, $\mathrm{CAL}$ (分子结构见参考文献[22])在苯(PCM 模型 ${ }^{[34,35]}$ )中 的吸收和发射光谱(如表 7 所示). 与实验值比较发现, 吸收光谱误差在 $15 \mathrm{~nm}$ 之内 $(\mathrm{AL}$ 和 NAL 吸收光谱误差 分别是 13.67 和 $15.02 \mathrm{~nm}$; 而 QAL 和 CAL 则分别是 0.97 和 $13.76 \mathrm{~nm}$ ). 计算的发射光谱与实验值比较发现, 误差 在 $30 \mathrm{~nm}$ 之内 $(\mathrm{AL} 、 \mathrm{QAL}$ 和 NAL 的计算值分别比实验 值小 23.72、17.68 和 $15.03 \mathrm{~nm}$, 而 CAL 则大 $29.48 \mathrm{~nm}$ ). 参考当前 DFT 可选泛函的计算精度, 所有计算值均比 较接近实验值, 也就是说, MPW3PBE/6-31+ $\mathrm{G}(d)$ 方法 比较适合我们的体系. 所有计算均采用 Gaussian09 程序 包完成 ${ }^{[36]}$.

辅助材料(Supporting Information) 所研究体系的基 态和激发态几何结构的笛卡尔坐标. 这些材料可以免费 从本刊网站(http://sioc-journal.cn/)上下载.

表 6 所研究化合物的发射波长、振子强度 $(f)$ 主要跃迁轨道及跃迁系数

Table 6 Emission wavelength, oscillator strength $(f)$, dominant transition orbitals, coefficient the studied compounds

\begin{tabular}{cccl}
\hline Compd. & Wavelength $/ \mathrm{nm}$ & $f$ & \multicolumn{1}{c}{ Main configuration } \\
\hline AOL & 432.39 & 0.4132 & HOMO $-1 \rightarrow$ LUMO $(-0.13006)$ \\
BEAOL & 559.13 & 0.0105 & HOMO $\rightarrow$ LUMO $(-0.69161)$ \\
BIAOL & 512.59 & 0.0000 & HOMO $\rightarrow$ LUMO $(-0.70571)$ \\
BPAOL & 516.38 & 0.0095 & HOMO $\rightarrow$ LUMO $(-0.70479)$ \\
PHAOL & 419.97 & 0.5369 & HOMO $\rightarrow$ LUMO $(0.70475)$ \\
PMAOL & 519.85 & 0.0000 & HOMO $-1 \rightarrow$ LUMO $+1(0.12165)$ \\
FLAOL & 420.72 & 0.7488 & HOMO $\rightarrow$ LUMO $(0.69496)$ \\
COAOL & 462.22 & 0.4895 & HOMO $\rightarrow$ LUMO $(-0.70216)$ \\
& & HOMO $-1 \rightarrow \operatorname{LUMO}(0.70410)$ \\
\hline
\end{tabular}


表 $7 \mathrm{AL}, \mathrm{QAL}, \mathrm{NAL}$ 和 CAL 在苯中的吸收和发射光谱 $(\mathrm{nm})$ 、振子强度 $(f)$

Table 7 Absorption and emission wavelength (nm), oscillator strength $(f)$ for AL, QAL, NAL, CAL in benzene

\begin{tabular}{cccccccc}
\hline \multirow{2}{*}{ Compd. } & \multicolumn{3}{c}{ Absorption spectrum } & & \multicolumn{3}{c}{ Emission spectrum } \\
\cline { 2 - 4 } \cline { 6 - 8 } & Wavelength & $f$ & Experiment $^{[22]}$ & & Wavelength & $f$ & Experiment $^{[22]}$ \\
\hline AL & 369.67 & 0.6108 & 356 & & 413.28 & 0.6402 & 437 \\
QAL & 357.03 & 0.3689 & 358 & & 405.32 & 0.3294 & 423 \\
NAL & 342.02 & 0.5036 & 327 & & 384.97 & 0.4764 & 400 \\
CAL & 382.24 & 0.4288 & 396 & & 498.48 & 0.0459 & 469 \\
\hline
\end{tabular}

\section{References}

[1] Shen, P. Y.; Wu, S. H.; Huang, Y. T.; Wei, Y. Res. Chem. Intermed. 2014, 40, 2199.

[2] Choi, A. Y.; Yamaguchi, T.; Han, C.-H. Res. Chem. Intermed. 2013, 39, 1571.

[3] Han, L. Z.; Wang, Z.; Hua, Y. J.; Ren, A. M.; Liu, Y. L.; Liu, P. J. Acta Chim. Sinica 2012, 70, 579 (in Chinese).

(韩立志, 王卓, 华英杰, 任爱民, 刘艳玲, 刘朋军, 化学学报, 2012, 70, 579.)

[4] Zhao, L. L.; Jiu, Y. D.; Wang, J. Y.; Zhang, X. W.; Lai, W. Y.; Huang, W. Acta Chim. Sinica 2013, 71, 1248 (in Chinese). (赵玲玲, 酒元达, 王建云, 张新稳, 赖文勇, 黄维, 化学学报, 2013, 71, 1248.)

[5] Huang, B.; Tang, J. N.; Jiang, W.; Yang, W.; Ban, X. X.; Sun, Y. M. Chin. J. Org. Chem. 2013, 33, 1395 (in Chinese).

(黄斌, 唐雯楠, 蒋伟, 杨文, 班金金金, 孙岳明, 有机化学, 2013, 33, 1395.)

[6] Wang, W. Y.; Ma, N. N.; Sun, S. L.; Qiu, Y. Q. Organometallics 2014, 33, 3341

[7] Song, H. J.; Zhang, M. Y.; Yu, H. L.; Wang, C. H.; Zou, H. Y.; Ma, N. N.; Qiu, Y. Q. Comput. Theor. Chem. 2014, 1031, 7.

[8] Gong, Y.; Zhang, Y.; Yuan, W. Z.; Sun, J. Z.; Zhang, Y. J. Phys. Chem. C 2014, 118, 10998.

[9] Shirota, Y. J. Mater. Chem. 2005, 15, 75.

[10] Shen, X. Y.; Yuan, W. Z.; Liu, Y.; Zhao, Q.; Lu, P.; Ma, Y.; Williams, I. D.; Qin, A.; Sun, J. Z.; Tang, B. Z. J. Phys. Chem. C 2012, $116,10541$.

[11] Shen, X. Y.; Wang, Y. J.; Zhao, E.; Yuan, W. Z.; Liu, Y.; Lu, P.; Qin, A.; Ma, Y.; Sun, J. Z.; Tang, B. Z. J. Phys. Chem. C 2013, $117,7334$.

[12] Kim, F. S.; Guo, X.; Watson, M. D.; Jenekhe, S. Adv. Mater. 2010, $22,478$.

[13] Lin, T.-C.; He, G. S.; Zheng, Q.; Prasad, P. N. J. Mater. Chem. 2006, 16, 2490.

[14] Grabowski, Z. R.; Rotkiewicz, K.; Rettig, W. Chem. Rev. 2003, $103,3899$.

[15] Liu, Y. J.; Vico, L. D.; Lindh, R. J. Photochem. Photobiol. A: Chem. 2008, 194, 261

[16] Cai, D.; Marques, M. A. L.; Milne, B. F.; Nogueira, F. J. Phys. Chem. Lett. 2010, 1, 2781.

[17] Ando, Y.; Niwa, K.; Yamada, N.; Enomoto, T.; Irie, T.; Kubota, H.; Ohmiya, Y.; Akiyama, H. Nat. Photonics 2008, $2,44$.

[18] Min, C. G.; Leng, Y.; Yang, X. K.; Huang, S. J.; Ren, A. M. Chem. J. Chin. Univ. 2014, 35, 564 (in Chinese). (闵春刚, 冷艳, 杨喜昆, 黄绍军, 任爱民, 高等学校化学学报, 2014, 35, 564.)

[19] Ishida, A.; Yoshikawa, T.; Kamidate, T. Anal. Biochem. 2003, 316, 127.

[20] Branchini, B. R.; Ablamsky, D. M.; Rosenman, J. M.; Uzasci, L.; Southworth, T. L.; Zimmer, M. Biochemistry 2007, 46, 13847.

[21] Viviani, V. R.; Oehlmeyer, T. L.; Arnoldi, F. G. C.; Brochet-
to-Braga, M. R. Photochem. Photobiol. 2005, 81, 843.

[22] Takakura, H.; Sasakura, K.; Ueno, T.; Urano, Y.; Terai, T.; Hanaoka, K.; Tsuboi, T.; Nagano, T. Chem. Asian J. 2010, 5, 2053.

[23] Li, Z. S.; Min, C. G.; Ren, A. M.; Zou, L. Y.; Xu, Z. J. Photochem. Photobio. A 2012, 243, 7-16

[24] Chu, T. Y.; Ho, M. H.; Chen, J. F.; Chen, C. H. Chem. Phys. Lett. 2005, 415, 137.

[25] Li, X.; Wang, X.; Gao, J.; Yu, X.; Wang H. Chem. Phys., 2006, $326,390$.

[26] The electron and hole transporting properties are related to the energies of HOMO and LUMO, IP(a) and EA(a) values as organic light-emitting diodes materials. In this paper, the HOMO and LUMO energies, IP(a) and EA(a) values of the studied molecules are compared with the vales of BNPB molecule in reference 25 . B3LYP/6-31G(d) method was used in reference 25 , and MPW3PBE/6-31+G(d) method is use in this paper. In fact, the difference in functional and basis sets may lead to different computational results. Therefore, the HOMO and LUMO energies, IP(a) and EA(a) values for BNPB were recalculation by MPW3PBE/ $6-31+\mathrm{G}(\mathrm{d})$ method.

[27] Nelsen, S. F.; Blomgren. F. J. J. Org. Chem., 2001, 66, 6551.

[28] Hutchison, G. R.; Ratner, M. A.; Marks, T. J. J. Am. Chem. Soc. 2005, 127, 2339.

[29] Perdew, J. P.; Burke, K.; Ernzerhof, M. Phys. Rev. Lett. 1996, 77, 3865.

[30] Adamo, C.; Barone, V. J. Chem. Phys. 1998, 108, 664

[31] Min, C. G.; Leng, Y.; Yang, X. K.; Ren, A. M.; Cui, X. Y.; Xu, M. L.; Wang, S. H. Chem. Res. Chin. Univ. 2013, 29, 982.

[32] Ren, X. F.; Ren, A. M.; Feng, J. K.; Sun, C. C. J. Photochem. Photobiol. A: Chem. 2009, 203, 92.

[33] Liu, Y. L.; Feng, J. K.; Ren, A. M. J. Comput. Chem. 2007, 28, 2500.

[34] Tomasi, J.; Mennucci, B.; Cammi, R. Chem. Rev. 2005, 105, 2999.

[35] Cramer, C. J.; Truhlar, D. G. Chem. Rev. 1999, 99, 2161.

[36] Frisch, M. J.; Trucks, G. W.; Schlegel, H. B.; Scuseria, G. E.; Robb, M. A.; Cheeseman, J. R.; Scalmani, G.; Barone, V.; Mennucci, B.; Petersson, G. A.; Nakatsuji, H.; Caricato, M.; Li, X.; Hratchian, H. P.; Izmaylov, A. F.; Bloino, J.; Zheng, G.; Sonnenberg, J. L.; Hada, M.; Ehara, M.; Toyota, K.; Fukuda, R.; Hasegawa, J.; Ishida, M.; Nakajima, T.; Honda, Y.; Kitao, O.; Nakai, H.; Vreven, T.; Montgomery, J. A. Jr.; Peralta, J. E.; Ogliaro, F.; Bearpark, M.; Heyd, J. J.; Brothers, E.; Kudin, K. N.; Staroverov, V. N.; Kobayashi, R.; Normand, J.; Raghavachari, K.; Rendell, A.; Burant, J. C.; Iyengar, S. S.; Tomasi, J.; Cossi, M.; Rega, N.; Millam, J. M.; Klene, M.; Knox, J. E.; Cross, J. B.; Bakken, V.; Adamo, C.; Jaramillo, J.; Gomperts, R.; Stratmann, R. E.; Yazyev, O.; Austin, A. J.; Cammi, R.; Pomelli, C.; Ochterski, J. W.; Martin, R. L.; Morokuma, K.; Zakrzewski, V. G.; Voth, G. A.; Salvador, P.; Dannenberg, J. J.; Dapprich, S.; Daniels, A. D.; Farkas, ö.; Foresman, J. B.; Ortiz, J. V.; Cioslowski, J.; Fox, D. J. Gaussian 09, Revision A.02, Gaussian Inc., Wallingford CT, 2009.

(Li, L.; Lu, Z.) 